requires no such process because it assumes that the $\mathrm{x}$-point is only intermittently present. By combining an analytical procedure for extrapolation of the surface magnetic field with high-resolution observations of the actual magnetic field, the Anglo-American team has shown that collisions of numerous tiny magnetic regions on the solar surface sometimes produce a short-lived $x$-point just above the Sun's surface. In previous work ${ }^{4}$, some of these magnetic regions, normally referred to as 'ephemeral active regions', have been observed to approach each other and disappear. Although the disappearance of the ephemeral regions is itself fairly slow and steady, it can nevertheless cause an $x$-point to pop out of the solar surface, move upwards a short distance into the atmosphere, and then drop back into the surface in a matter of minutes.

Additional support for the new model comes from very-high-resolution images of X-ray bright points obtained with an advanced technology X-ray telescope during a rocket launch at White Sands, New Mexico, in July 1991 (ref. 5). One of these images is shown in the figure on the previous page, together with a blow-up of one of the bright-point sources. The expanded image shows thin filaments of superheated gas extending outwards from a central region. These filaments are neatly explained by the new model as lying along magnetic lines of force which are directly connected to the $\mathrm{x}$-points. So the new model successfully accounts for the fine structure, as well as the intermittency, of the X-ray bright points.

What is needed to confirm this new model is numerical simulation of the detailed plasma dynamics as the x-point appears and disappears. Coupled with higher-resolution images at different $\mathrm{X}$-ray energies, such simulations should make it possible to prove or disprove this concept.

$T$. G. Forbes is in the Institute for the Study of Earth, Oceans, and Space at the University of New Hampshire, Durham, New Hampshire 03847, USA.

\footnotetext{
Priest. E. R. Parnell, C. E. \& Martin, S. F. Astrophys. 427.459 (1994)

427. 459 (1994).
Parnelt. C. E. Priest, E. R. \& Golub. L. Solar Phys. (in the press).

Dungey, J. W. Phil. Mag. 44, 725 (1953).

Martin. S. F. \& Harvey, K. L. Solar Phys. 64, 93 (1979)

Golub, L. Zirin \& Wang, H. Astrophys. J. (in the press)
}

\section{Call of the wild}

THE skulls of Arctic wolves became smaller and altered in shape between 1930 and 1950. J. Clutton-Brock et al. (J. Zool., Lond. 233, 19-36; 1994) came to this conclusion after making a detailed series of measurements on museum specimens, and then asked "Why?". After mulling over various explanations, they conclude that the change in skull morphology was probably due to a burst of hybridization with huskies. Wolf skulls from the period 1930-50 are similar to that of a known wolf-husky hybrid; and the authors argue that, with the advent of the motor-sledge, numbers of huskies were made redundant and took to the wild - there to mate with wolves.

\section{Pair shaped}

DESIGNINGa molecular receptor that will pick a particular cation species out of solution is a chemist's favourite pastime: the trick is to synthesize a molecule whose nucleophilic binding sites complement the chosen cation. The molecule here, synthesized by D. M Rudkevich et al., goes one better: it

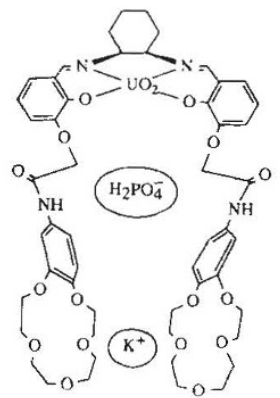

selects both halves of the biologically useful salt $\mathrm{KH}_{2} \mathrm{PO}_{4}$, the crown ether jaws grasping the $\mathrm{K}^{+}$cation whilst the central cleft binds an $\mathrm{H}_{2} \mathrm{PO}_{4}{ }^{-}$anion (Angew. Chem. int. Edn Engl. 33, 467-468; 1994). The hope is that receptors like this will be good for selective transport across membranes and in sensor technology.

\section{Lend a hand}

D. PURves and colleagues have come up with another puzzle for those exercised by the matter of right-and left-handedness. Exploiting Archimedes' principle, they have measured the hand size of groups of both right- and left-handers by volumetric displacement; that is, volunteers submerged their hands in a tank of water up to a defined point, and the amount of water displaced was weighed. Purves et al. find that whereas there is a clear asymmetry in hand size among their righthanded subjects, in whom the right hand is larger than the left, the corresponding difference is much less marked in lefthanders (Proc. natn. Acad. Sci. U.S.A. 91 5030-5032; 1994). The underlying research agenda is neurobiological: the authors intend to go on and explore whether hand asymmetry or lack of it is also evident in the related neural structures. provoke neural induction, and interest in the problem waned because it was felt that it would not be possible to pinpoint a specific substance as responsible for such The older work was done with newt embryos, whereas the recent renaissance stead the African clawed frog Xenopus laevis. So obsessed were we with the idea for some time nobody worked on it induction and dorsalization, people in 作 gradually dawned on us that we ization; rather, we were not seeing it at all. lem after all. In fact the absence of candidate substances seemed more of a problem. ments that would neuralize Xenop ectoderm were the protein kinase $\mathrm{C}$ agonist $\mathrm{TPA}^{4}$, whose effects are somewhat marginal, and cellular disaggregation ${ }^{5}$ which works very well but was suspected of being some sort of artefact. Neuralization of disaggregated cells occurs at low density but is suppressed at high density, and we suggested at the time that it might be due to the dilution of an endogenous 